\title{
Rapid Neuroinflammatory Response Localized to Injured Neurons After Diffuse Traumatic Brain Injury in Swine
}

\author{
Kathryn L. Wofford ${ }^{\text {a, b, c }}$, James P. Harris ${ }^{\text {a, c }}$, Kevin D. Browne ${ }^{\text {a, c }}$, Daniel P. Brown ${ }^{\text {a, c }}$, \\ Michael R. Grovola ${ }^{\text {a, }}$, Constance J. Mietus ${ }^{\text {c }}$, John A. Wolf ${ }^{\text {a, c }}$, John E. Duda ${ }^{\text {a, d }}$, Mary E. \\ Putt $^{\text {e }}$, Kara L. Spiller ${ }^{\text {b }}$, D. Kacy Cullen ${ }^{\text {a, c, * }}$
}

${ }^{a}$ Center for Neurotrauma, Neurodegeneration \& Restoration, Corporal Michael J. Crescenz Veterans Affairs Medical Center, 3900 Woodland Avenue, Philadelphia, PA, 19104, USA.

${ }^{b}$ School of Biomedical Engineering, Science and Health Systems, Drexel University, 3141 Chestnut Street, Philadelphia, PA, 19104. USA.

${ }^{c}$ Center for Brain Injury \& Repair, Department of Neurosurgery, University of Pennsylvania, 105 Hayden Hall, 3320 Smith Walk, Philadelphia, PA, 19104, USA.

${ }^{d}$ Department of Neurology, Corporal Michael J. Crescenz Veterans Affairs Medical Center, 3900 Woodland Avenue, Philadelphia, PA, 19104, USA.

${ }^{e}$ Department of Biostatistics and Epidemiology, Hospital of the University of Pennsylvania, 621 Blockley Hall, 423 Guardian Drive, Philadelphia, PA, 19104, USA.

* Correspondence and reprint request should be directed to:

D. Kacy Cullen (dkacy@mail.med.upenn.edu), Department of Neurosurgery, University of Pennsylvania, 105 Hayden Hall, 3320 Smith Walk, Philadelphia, PA, 19104, USA. Phone: (215) 7468176. Fax: (215) 573-3808 


\section{Author Addresses:}

Kathryn L. Wofford (kate.wofford@drexel.edu)

James P. Harris (jph18@case.edu)

Kevin D. Browne (kbrowne@ mail.med.upenn.edu)

Daniel P. Brown (dbrow@ mail.med.upenn.edu)

Michael R. Grovola (mgrovola@mail.med.upenn.edu)

Constance J. Mietus (mietusc@ gmail.com)

John A. Wolf (wolfjo@upenn.edu)

John E. Duda (john.duda@va.gov)

Mary E. Putt (mputt@ mail.med.upenn.edu)

Kara L. Spiller (kls35@drexel.edu)

D. Kacy Cullen (dkacy@mail.med.upenn.edu) 


\section{Abstract}

Despite increasing appreciation of the critical role that neuroinflammatory pathways play in brain injury and neurodegeneration, little is known about acute microglial reactivity following diffuse traumatic brain injury (TBI) - the most common clinical presentation that includes all concussions. Therefore, we investigated acute microglial reactivity using a porcine model of closed-head rotational velocity/acceleration-induced TBI that closely mimics the biomechanical etiology of inertial TBI in humans. We observed rapid microglial reactivity within 15 minutes of both mild and severe TBI. Strikingly, microglial activation was restrained to regions proximal to individual injured neurons - as denoted by trauma-induced plasma membrane disruption - which served as epicenters of acute reactivity. Single-cell quantitative analysis showed that in areas free of traumatically permeabilized neurons, microglial density and morphology were similar between sham or following mild or severe TBI. However, microglia density increased and morphology shifted to become more reactive in proximity to injured neurons. Microglial reactivity around injured neurons was exacerbated following repetitive TBI, suggesting further amplification of acute neuroinflammatory responses. These results indicate that neuronal trauma rapidly activates microglia in a highly localized manner, and suggest that activated microglia may rapidly influence neuronal stability and/or pathophysiology after diffuse TBI.

Keywords: Neuroinflammation; Microglia Reactivity; Diffuse Traumatic Brain Injury; Permeabilized Neurons; Concussion

Abbreviations: TBI = traumatic brain injury; LY = Lucifer yellow; Iba1 = ionized calcium-binding adapter molecule 1; GFAP = glial fibrillary acidic protein 


\section{Introduction}

Traumatic brain injury (TBI) is a major health and socioeconomic problem, with over 1.7 million individuals afflicted each year and over 5 million exhibiting chronic neurological deficits in the U.S. alone (Faul M, Xu L, Wald MM, 2010; Hyder et al., 2007; Langlois et al., 2006). Even so-called "mild" TBI - otherwise known as concussion - may lead to cognitive disruptions immediately post-injury as well as persistent neurological deficits likely resulting from disruption of neuronal circuitry (De Kruijk et al., 2001; De Monte et al., 2006; Leininger et al., 1990; Wolf and Koch, 2016). However, it is not well understood how microglia, the inflammatory modulators of the CNS, contribute to acute neuronal health, homeostasis, and/or pathophysiology after TBI. Previously, murine CNS injury models indicated that microglia react to laser ablation within minutes of the initial insult (Davalos et al., 2005; Dibaj et al., 2010). It remains unclear if a similar phenomenon occurs following closed-head (i.e., non-penetrating) TBI generally resulting from falls, collisions and/or blunt impacts (Coronado et al., 2012; Faul M, Xu L, Wald MM, 2010; Langlois et al., 2006). Indeed, the vast majority of clinical TBIs are closed-head injuries caused by a sudden jolt or blow to the head, resulting in rapid rotational velocity/acceleration (inertial loading) and diffuse strain fields throughout the brain (Adams et al., 1989; Ommaya and Gennarelli, 1974; Povlishock, 1992; Smith and Meaney, 2000). These TBIs are difficult to model because there is no injury epicenter as is the case with predominantly focal TBIs - generally the dominant component of preclinical models using rodents - and the resulting neuropathology is often extremely subtle. Moreover, large brain mass and gyrencephalic architecture are key features in replicating the biomechanics and pathophysiological progression of closed-head TBI.

Therefore, we investigated acute microglial reactivity immediately following inertial TBI using an established porcine model with biomechanical fidelity to closed-head TBI in humans (Cullen et al., 2016; Meaney et al., 1995; Smith et al., 1997). Since diffuse TBIs by definition lack a focal injury epicenter, we explored microglial activation in the context of neuronal trauma, specifically breaches in plasma membrane integrity (Cullen et al., 2011; Geddes et al., 2003; LaPlaca et al., 2009; Singleton and Povlishock, 2004). Such plasma membrane disruptions are a hallmark consequence of supra-threshold loading during TBI, and may alter cell homeostasis due to loss of ionic gradients, osmotic imbalance, and calcium dysregulation (LaPlaca et al., 1997; Stone et al., 2004; Weber et al., 1999). These acute membrane breaches are generally transient, as initially compromised cells survive the insult but may be dysfunctional or undergo delayed cell death (Cullen et al., 2011; Farkas et al., 2006; Singleton and Povlishock, 2004; Whalen et al., 2008). In addition, microglia have been shown to be reactive at 
intermediate and chronic (e.g., days to years) time points after TBI (Johnson et al., 2013; Kelley et al., 2007; Lafrenaye et al., 2015), and glial activation is likely mediated by warning factors released from damaged neurons (Davalos et al., 2005; Lee, 2013). Thus we hypothesized that microglia would be most reactive, as measured by distribution and morphology, in regions exhibiting acute neuronal damage and correlating with injury severity and number of injuries. We tested this hypothesis by subjecting swine to single or repetitive closed-head rotational TBI at levels previously established to result in an injury phenotype consistent with clinical definitions of "mild" or "severe" TBI in humans (Browne et al., 2011; Cullen et al., 2016; Smith et al., 2000). In order to assess trauma-induced alterations in membrane permeability, the cell-impermeant dye Lucifer yellow (LY) was delivered into the ventricles to diffuse throughout the interstitial tissue prior to injury. LY would only gain intracellular access if the plasma membrane became compromised during or immediately following injury, similar in principle to other fluorescent permeability tracers used in the neurotrauma field (Cullen et al., 2011; Geddes et al., 2003; Harris, 2015; LaPlaca et al., 2009; Simon et al., 2009; Singleton and Povlishock, 2004; Stone et al., 2004). Following closed-head rotational TBI, microglial cell characteristics were quantified as a function of distance from injured neurons. This model presents a unique potential for analyzing acute microglia reactively following closed-head diffuse TBI, and our findings suggest that rapid microglia activation depends on proximity to traumatically permeabilized neurons.

\section{Materials And Methods}

\section{Animal Care and Anesthesia}

All procedures and protocols were approved by the Animal Care and Use Committee of the University of Pennsylvania. All animal care complied with the Guide for the Care and Use of Laboratory Animals (U.S. National Research Council, 1996) and followed the ARRIVE Guidelines. Female Yorkshire swine $(\mathrm{n}=19)$ with an average weight of $22.5 \mathrm{~kg}$ were utilized in this study. No special diets were provided as the animals were housed indoors with feed and water, ad libitum. The housing facility was accredited by the Association for Assessment and Accreditation of Laboratory Animal Care International. Prior to the injury procedure, animals were fasted for 18-20 hours with water remaining ad libitum. After induction with a cocktail of ketamine (20-30mg/kg; Hospira, 0409-2051-05) and midazolam (0.4-0.6mg/kg; Hospira, 0409-2596-05), anesthesia was provided with 5\% isoflurane (Piramal, 66794-013-25) via a snout mask and glycopyrrolate was given $(0.01 \mathrm{mg} / \mathrm{kg}$; West-Ward Pharmaceutical Corp., 0143-9682-25) subcutaneously. The animal was then intubated, and isoflurane 
was connected at maintenance levels (1.5-2.0\%). Eye lubricant was used to minimize drying. Physiological monitoring allowed titration of anesthesia so that $\mathrm{SpO} 2$, heart rate, and respirations were within acceptable ranges (97-100\% SpO2, HR between 110-130, and respirations between 9-12 breaths per minute, respectively).

\section{Delivery of Lucifer Yellow via Intracerebroventicular Injections}

LY (Invitrogen, L453, Carlsbad, CA) was delivered into the lateral ventricles of all animals before the rotational/sham injury to demarcate cells permeabilized during the rotational injury. This aldehyde-fixable, normally cell-impermeable dye was delivered via a Hamilton syringe (Reno, NV). Animals were placed in a stereotactic head frame. The scalp was shaved and swabbed with betadine before a $4 \mathrm{~cm}$ incision was made. The scalp was reflected from the skull in order to visualize Bregma. Two $5 \mathrm{~mm}$ craniectomies were made at the following stereotactic co-ordinates: $1.0 \mathrm{~mm}$ posterior to Bregma, $\pm 6.0 \mathrm{~mm}$ lateral. A small incision in the dura was made with a 19-gauge needle. For each site in serial fashion, a sterile Hamilton syringe was lowered $18.0 \mathrm{~mm}$ from the surface of the dura mater to access the ventricles. Tissue was left to stabilize for 2 minutes before delivering $500 \mu \mathrm{L}$ of LY $(0.4 \mathrm{mg} / \mathrm{kg}$ in sterile saline) over a period of 10 minutes on each side using an UltraMicroPump III (World Precision Instruments, Sarasota, FL). The needle was slowly withdrawn at a rate of 2 $\mathrm{mm} / \mathrm{minute}$, and the burr holes were sealed with bone wax. The incision site was sutured with $0-0$ sutures, and the area was swabbed with betadine. A surgical plane of anesthesia was maintained through the injury/sham procedure and sacrifice. The injury occurred 2 hours after the start of the first injection, which was optimized to allow even LY distribution throughout the interstitial tissue.

\section{Porcine Closed-Head Rotational/Inertial TBI}

Closed-head rotational TBI was induced under anesthesia using the HYGE pneumatic actuator, a device capable of producing pure impulsive non-impact head rotation with a controlled relationship between maximum rotational acceleration and injury severity, as previously described (Browne et al., 2011; Cullen et al., 2016; Smith et al., 2000, 1997). The well-characterized model rapidly accelerates the head and induces inertial forces representative of human TBI from falls, collisions, or blunt impacts. Angular velocity was recorded using a magneto-hydrodynamic sensor (Applied Technology Associates, Albuquerque, NM) connected to a National Instruments DAQ, controlled by LabVIEW. Briefly, the animal was randomly assigned to an injury group and the animal's head was secured to a padded bite plate under anesthesia, and mounted to the HYGE device. In the miniature pigs, sagittal plane head accelerations (transverse to the brainstem) cause an enhancement of strain in the brainstem region 
compared to coronal plane accelerations (circumferential to the brainstem), therefore reduced levels of angular velocity/acceleration are required to elicit loss of consciousness and coma (Browne et al., 2011; Cullen et al., 2016; Meaney et al., 1995; Smith et al., 2000, 1997). Therefore, to induce a "mild" injury phenotype, single rapid head rotation was performed in the coronal plane (peak angular velocities of 193-299 radians/second; $\mathrm{n}=7$ ), whereas to induce a moderate-to-severe injury phenotype rotation occurred in the sagittal plane (peak angular velocities: 80-139 rad/s; n=5). In prior studies, it was empirically determined that head rotation at these levels of angular velocity/acceleration in the coronal plane did not induce measurable loss of consciousness or hematoma. However, head rotation at these levels of angular velocity/acceleration in the sagittal plane induced prolonged loss of consciousness and often significant hematoma (Cullen et al., 2016). In addition to the single injuries, we performed repetitive injuries on a small cohort of animals in both the sagittal and coronal planes. Repetitive head rotation was either performed on the same day (separated by 15 minutes) or separated by 7 days; these animals only received LY intracerebroventicular injection on the day of their second injury ( $\mathrm{n}=4$ total). Sham animals received all other procedures absent head rotation $(n=3)$.

\section{Brain Tissue Acquisition and Processing}

All animals were sacrificed within 15 minutes of head rotational injury (15 minutes following the second injury for the animals subjected to repeated head rotation). Animals were transcardially perfused using heparinized saline $\left(4^{\circ} \mathrm{C}, 4\right.$ liters $)$ followed by chilled, $4 \%$ paraformaldehyde $\left(4^{\circ} \mathrm{C}, 8-10\right.$ liters; Sigma, P6148). Brains were blocked in the coronal plane at $5 \mathrm{~mm}$ intervals, then cleared in PBS (phosphate buffered saline) and transferred to $30 \%$ sucrose until they sunk in the solution. The blocks were placed in OCT, flash frozen, and stored at $-80{ }^{\circ} \mathrm{C}$. Twenty-micron thick sections were cut coronally on a cryostat (Leica CM1860).

\section{Immunohistochemistry and Imaging}

Florescent immunohistochemistry was performed with antibodies against ionized calciumbinding adapter molecule 1 (Iba1; rabbit IgG 1:300; Wako, 019-19741) or glial fibrillary acidic protein (GFAP; rabbit IgG 1:500; Millipore AB5804). Mounted sections were washed in PBS, and blocked in $4 \%$ non-specific goat serum with $0.3 \%$ Triton-X for 1 hour at room temperature. Slices were incubated with primary antibody in blocking solution overnight at $4^{\circ} \mathrm{C}$. Anti-rabbit secondary antibody (Invitrogen, A31573) was applied at a 1:1000 concentration for 2 hours at room temperature in blocking solution. Sections were counterstained with Hoechst (Hoechst 33342, Life Technologies, H3570) or Fluo-Nissl (N21479, Life Technologies). Sections were preserved with Fluoromount-G (Southern 
Biotech) and coverslipped. Immunohistochemical images were acquired with a confocal microscope (Nikon A1RSI Laser Scanning Confocal or Zeiss LSM 710) on the 10x or 20x objectives.

\section{Quantification of Ibal Microglia Morphology and Distribution}

While all animals in this study were qualitatively evaluated for changes in microglial reactivity, a subset of animals were subjected to in-depth single-cell analyses involving detailed quantitative scoring of microglial distribution and morphology in relation to permeabilized neurons $(8,495$ total microglia scored across nine animals). An average of 1,000 Iba1-labeled microglia were analyzed within each animal $(1,177.5 \pm 90.3$ cells for injured animals and $476.7 \pm 44.9$ cells for sham animals) to investigate morphological and density differences in sham $(\mathrm{n}=3$ ), mild (coronal plane rotation at 207-220 rad/sec; $\mathrm{n}=3$ ), and severe (sagittal plane rotation at $114-139 \mathrm{rad} / \mathrm{sec} ; \mathrm{n}=3$ ) injury animals. Note that the quantitative analyses were performed on animals that experienced a lower rotational velocity/acceleration within the coronal plane (approximating loading levels found to induce concussion in humans based on biomechanical scaling) to generate mild-TBI whereas quantitative analyses were performed on animals that experienced a higher kinematic loading (thus more severe trauma) within the sagittal plane. Images of microglia localized with $\mathrm{LY}^{+}$neurons and internal control images of microglia not localized with $\mathrm{LY}^{+}$neurons were generated for quantification. All $\mathrm{Iba}^{+} / \mathrm{Hoechst}^{+}$microglia were morphologically scored in ImageJ using a straight-line tool to measure the total cell diameter at the widest points and the soma diameter at the widest points. Thereafter, the cell's total diameter was divided by the soma diameter to generate a ratio where higher ratios represent a ramified, scanning morphology (Fig. 1A) and lower ratios represent a reactive, amoeboid morphology (Fig. 1B). This morphological scoring methodology was inspired by Hovens et al. (2015), which similarly utilized a cell body to cell soma ratio, but for a population of microglia; we adapted this approach to allow single cell analysis. This scoring procedure was subsequently validated against the Hovens et al. morphology scoring method using a linear regression (Hovens et al., 2014) (Fig. 1D) executed in Prism Version 6 for Mac OS X.

In order to determine microglia's morphology as a function of distance from the nearest $\mathrm{LY}^{+}$ neuron, images of microglia localized with $\mathrm{LY}^{+}$neurons were binned into three regions: a proximal region, where distance (d) from a permeabilized neuron was less than or equal to $60 \mu \mathrm{m}$; a medial region where $60<\mathrm{d} \leq 105 \mu \mathrm{m}$; and a distal region where $\mathrm{d}>105 \mu \mathrm{m}$ from $\mathrm{LY}^{+}$neurons (Fig. 1C). Bin sizes were chosen so that proximal and medial regions would be approximately four times the width of a microglia soma. Microglia measurements from each region were pooled to determine how distance from the nearest $\mathrm{LY}^{+}$neuron affected microglia morphology. If microglia were on the boundary of two 
regions they were grouped in the more proximal zone. Microglia morphology data from Figure 5 is broken down to illustrate individual animal trends in Supplementary Figure 2. Microglia density as a function of distances was calculated by counting the number of microglia morphology tracings in each region before normalizing to the total area of each respective region. In order to examine the changing trend of microglia density across different regions, relative frequency graphs were smoothed utilizing a second-order smoothing function considering four neighboring points (Prism Version 6; Fig. 4G-H). Subsequent, non-smoothed relative frequency graphs of microglia morphology scores were plotted by region and injury for trend comparisons within each animal (Supplementary Fig. 1). All data is presented as mean \pm standard error of the mean, where mean is calculated based on the number of animals and standard error of the mean was calculated as the standard deviation divided by the square root of the number of animals.
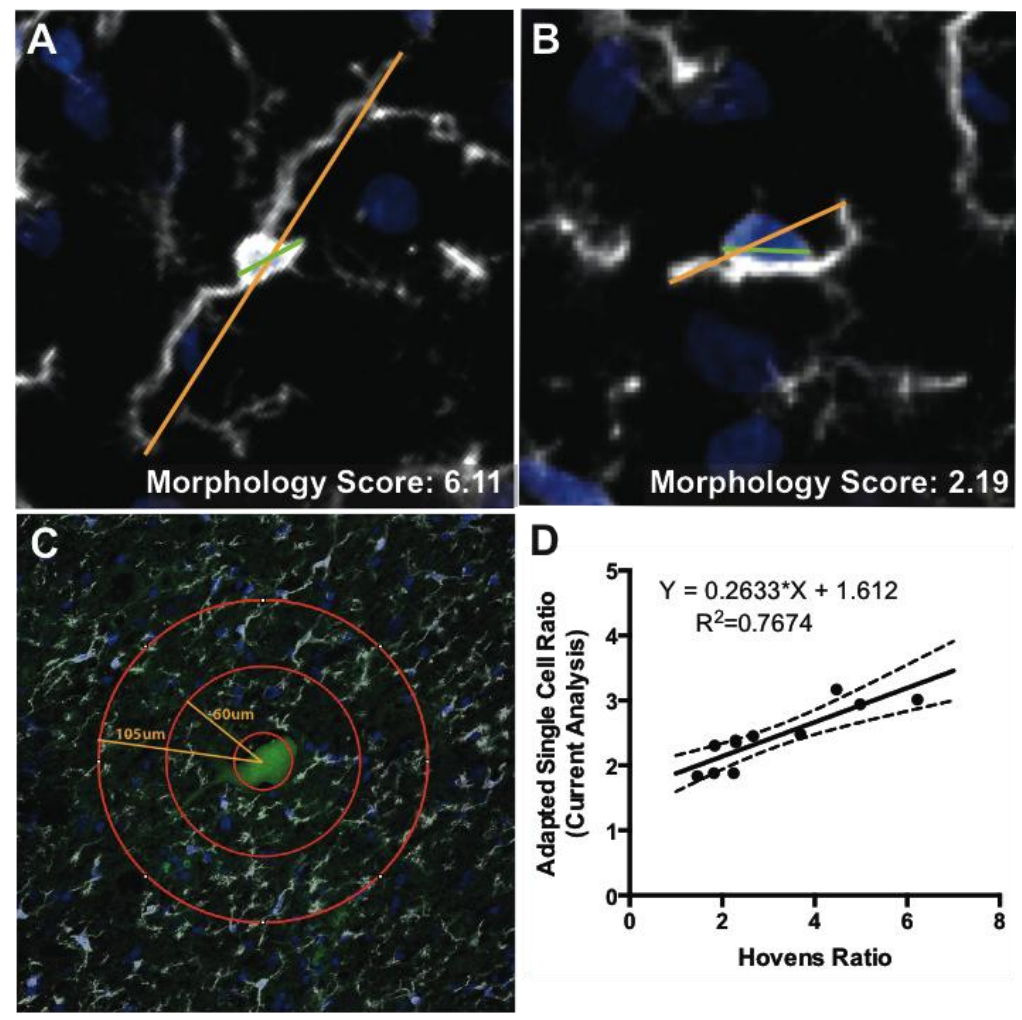

Figure 1. Method associated with microglial (Iba1 ${ }^{+}$, white) morphology and distribution quantification. Cell diameter (orange line) and the soma diameter (green line) were measured with a straight-line tool to generate a morphology score with higher values representing a more ramified morphology and values closer to one representing a more reactive morphology (A-B). To quantify morphology as a function of distance from permeabilized neurons, concentric circles were generated around LY neurons, creating proximal (less than $60 \mu \mathrm{m}$ ), medial (between 60 and $105 \mu \mathrm{m}$ ), and distal (greater than $105 \mu \mathrm{m})$ zones of microglia (C). This nascent single cell 
morphology characterization methodology was compared to an established microglia morphology metric (Hovens et al., 2014) with a linear regression and 95\% confidence interval bands in order to validate this new methodology ( $\mathrm{D}$; using 11 images each containing 10-23 microglia). The linear regression had an R-squared value of 0.7674 .

\section{Statistical Analysis}

To achieve approximate normality, the microglia morphology scores and density data were logtransformed. A priori, it was of interest to determine (1) whether mean microglial morphology or density differed by injury level and (2) whether, for each injury level, mean microglial morphology or density differed between regions. Because there were insufficient animals to formally test for an interaction between injury and regions, we considered these hypotheses separately. For the morphology scores, we fit a linear mixed effects model that included animal as a random effect nested within injury; for the cell density data, which had a more skewed distribution, we used a generalized estimating equation with a Poisson link, and adjusted for the size of the area examined. This modeling/testing procedure is similar to ANOVA but allows repeated measurements on the individual animals (Fitzmaurice et al., 2004). If the density or morphology test on the effect of region or injury achieved significance, we carried out a Bonferroni-Holm post-hoc test to adjust for multiple comparisons and maintain a family-wise error rate of 0.05 . We note that analyses of the injury effect contrasted different animals, while analyses of the region effect were based on within-animal contrasts. The within-animal contrast eliminates betweenanimal variability. Thus smaller mean differences are detectable for the analysis of differences between region, a within-animal contrast, than between injury levels, a between-animal contrast. The type I error rate was set to 0.05 and all hypothesis tests are two-sided. All analyses were carried out in R Studio Version 0.99.896 using R and the nlme, geepack, gtools, and gmodels packages (The R Development Core Team, 2016).

\section{Results}

All animals were euthanized 15 minutes after the last TBI, and their brains processed for immunohistological and confocal microscopy examination of plasmalemmal permeability and microglial activation. We found that the membrane impermeable dye, LY, demarcated neuronal somata and neurites experiencing acute membrane permeability as a result of diffuse TBI (Fig. 2), consistent with previous reports (LaPlaca et al., 2009; Simon et al., 2009). Using confocal double-labeled 
fluorescent micrographs we determined distribution and morphology of microglia, denoted by expression of $\mathrm{Iba1}^{+}$, as a function of distance from neurons exhibiting acute plasma membrane breaches (based on LY uptake) in both the cerebral cortex (cortical gray matter and sub-cortical white matter) (Fig. 3F-J, 4A-E, 5D-H, 6A-D) and hippocampus (Fig. 5A-C). Microglial activation was quantified using a cell body to cell size ratio (Fig. 1).

Sham-injured animals exhibited few, if any, $\mathrm{LY}^{+}$neurons, whereas both mild and severe TBI resulted in a marked increase in the density of $\mathrm{LY}^{+}$neurons. Moreover, the cumulative burden of permeabilized neurons was at least 6-fold greater after severe versus mild TBI (Fig. 3B, D). Across various anatomical levels of the cortex, the distribution of permeabilized neurons exhibited a "skip phenomenon" where localized patches of injured neurons were observed that were spanned by fields exhibiting no neuronal damage. Since injured neurons were the focal point for microglial analysis, fields were selected that contained permeabilized neurons (quantified regions contained a mean density of 18.2 $\pm 5.5 \mathrm{LY}^{+}$neurons $/ \mathrm{mm}^{2}$ in mild-TBI and $31.1 \pm 5.5 \mathrm{LY}^{+}$neurons $/ \mathrm{mm}^{2}$ in severe-TBI) and were compared to adjacent fields in the same animals that did not have $\mathrm{LY}^{+}$neurons as well as anatomically similar fields in sham animals. 


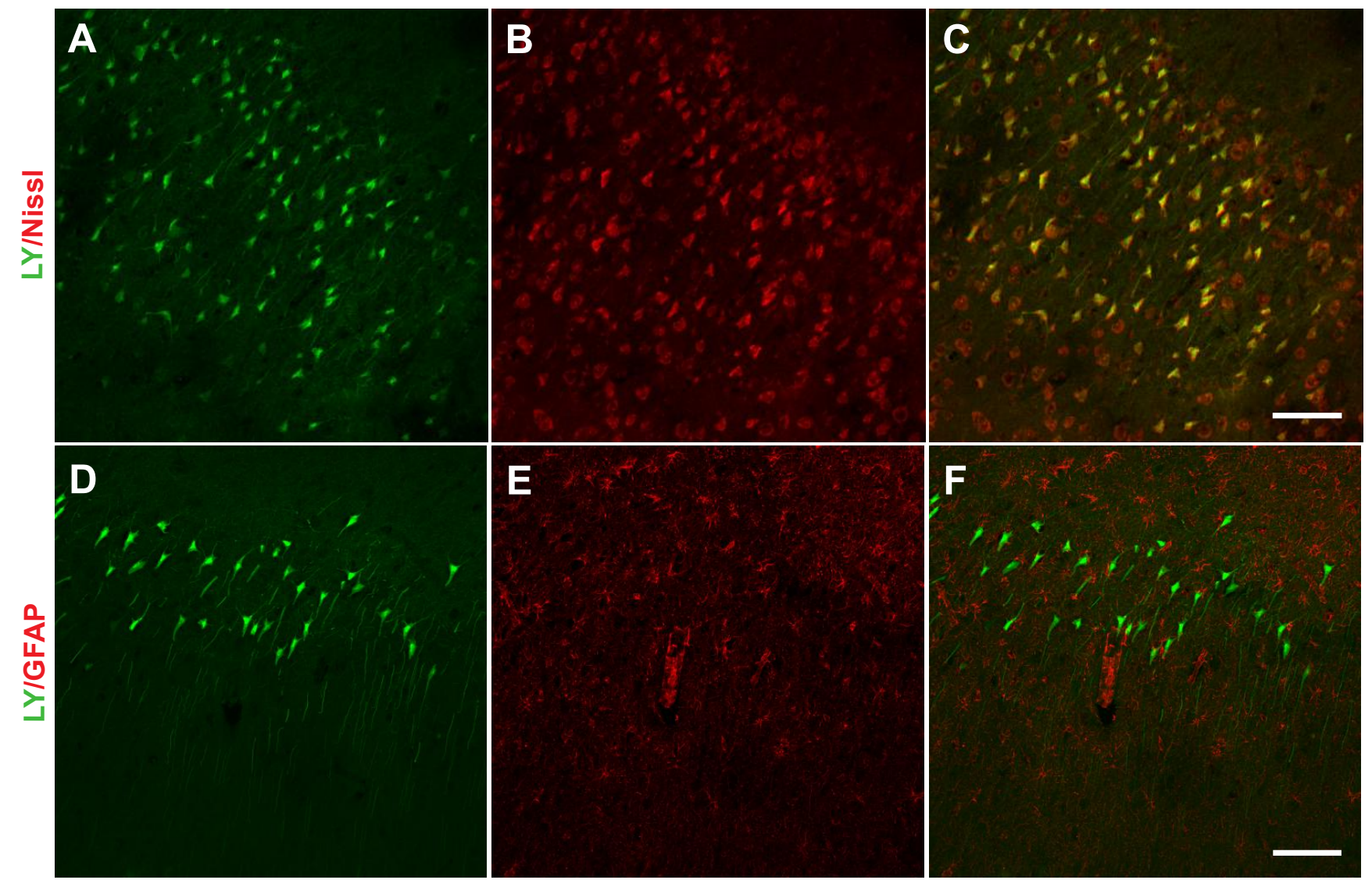

Figure 2. Lucifer yellow labeled damaged neurons but not astrocytes after TBI. Confocal micrographs demonstrating that $\mathrm{LY}^{+}$cells (green, A, D) colocalized with Nissl bodies (red, B), indicative of a neuronal phenotype, but did not colocalize with GFAP (red, E), an astrocytic marker, with overlay of each label $(\mathrm{C}, \mathrm{F}) . \mathrm{LY}^{+}$cells after TBI were also morphologically consistent with neurons. Thus, intraventricularly administered LY predominantly demarcated membrane-permeabilized neurons after TBI. Scale bars $=100 \mu \mathrm{m}$. 


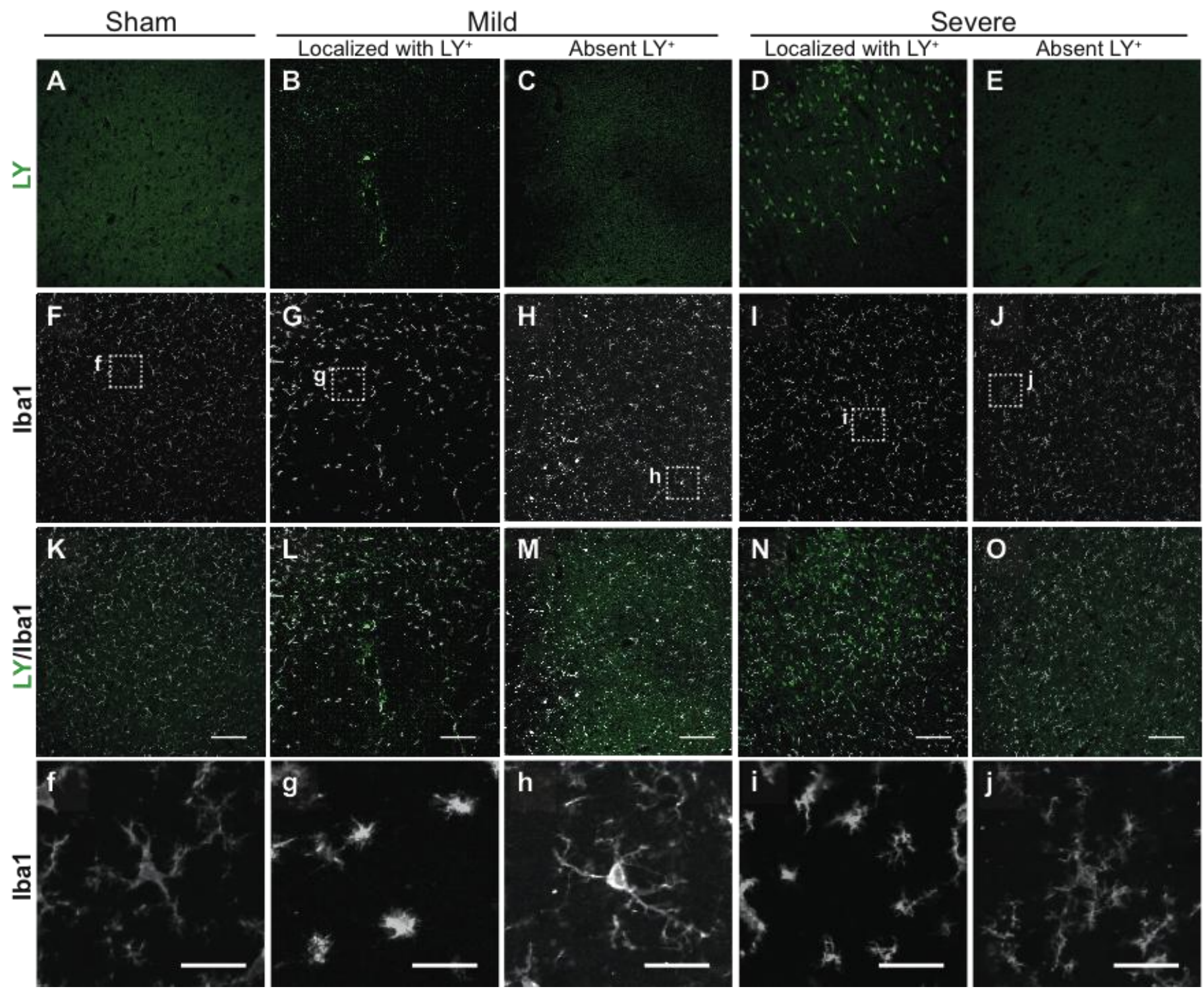

Figure 3. Regions of the cerebral cortex containing $\mathrm{LY}^{+}$neurons were co-localized with reactive microglia post-TBI. Micrographs of $\mathrm{LY}^{+}$neurons (green, A-E), Iba1 ${ }^{+}$microglia (white, F-J), overlay (K-O), and call-out boxes (f-j) after sham (A, F, K), mild (B-C, G-H, L-M), or severe (D-E, I-J, N-O) TBI. Images were either from regions localized with $\mathrm{LY}^{+}$neurons $(\mathrm{B}, \mathrm{D}$, $\mathrm{G}, \mathrm{I}, \mathrm{L}, \mathrm{N})$ or regions absent $\mathrm{LY}^{+}$neurons $(\mathrm{C}, \mathrm{E}, \mathrm{H}, \mathrm{J}, \mathrm{M}, \mathrm{O})$. Neurons exhibiting acute plasma membrane permeability $\left(\mathrm{LY}^{+}\right)$were appreciable following either mild or severe TBI, with approximately a 6-fold greater burden of $\mathrm{LY}^{+}$neurons following severe versus mild injury. Scale bars $=100 \mu \mathrm{m}(\mathrm{K}-\mathrm{O})$ or scale bars $=25 \mu \mathrm{m}(\mathrm{f}-\mathrm{j})$. 
In sham animals, we found minimal evidence of reactive microglia, whereas following mild or severe TBI, microglia in the cortex and hippocampus exhibited extensive reactive morphology in fields containing permeabilized neurons (Fig. 3F-J, 4A-E, 5A-H). To better characterize microglial activation on a single-cell level, we quantified the distribution and morphology of $\mathrm{Iba}^{+} / \mathrm{Hoechst}^{+}$microglia in confocal micrographs as a function of the distance from traumatically permeabilized neurons $(8,495$ microglia scored across multiple cortical regions from $n=9$ pigs). This analysis revealed that in areas absent permeabilized neurons, microglia distribution was equivalent across sham, mild, and severe injuries (Fig. 4F). In contrast, in areas that contained traumatically permeabilized neurons, there was a significant increase in microglia density that was enhanced based on proximity to injured neurons following either mild or severe TBI (Fig. 4F-H, Supplementary Fig. 1). Intriguingly, we found that regions absent $\mathrm{LY}^{+}$neurons did not differ from sham animals. Furthermore, we found that microglia density around $\mathrm{LY}^{+}$neurons after severe injures was comparable to microglia density around $\mathrm{LY}^{+}$ neurons after mild injuries. 

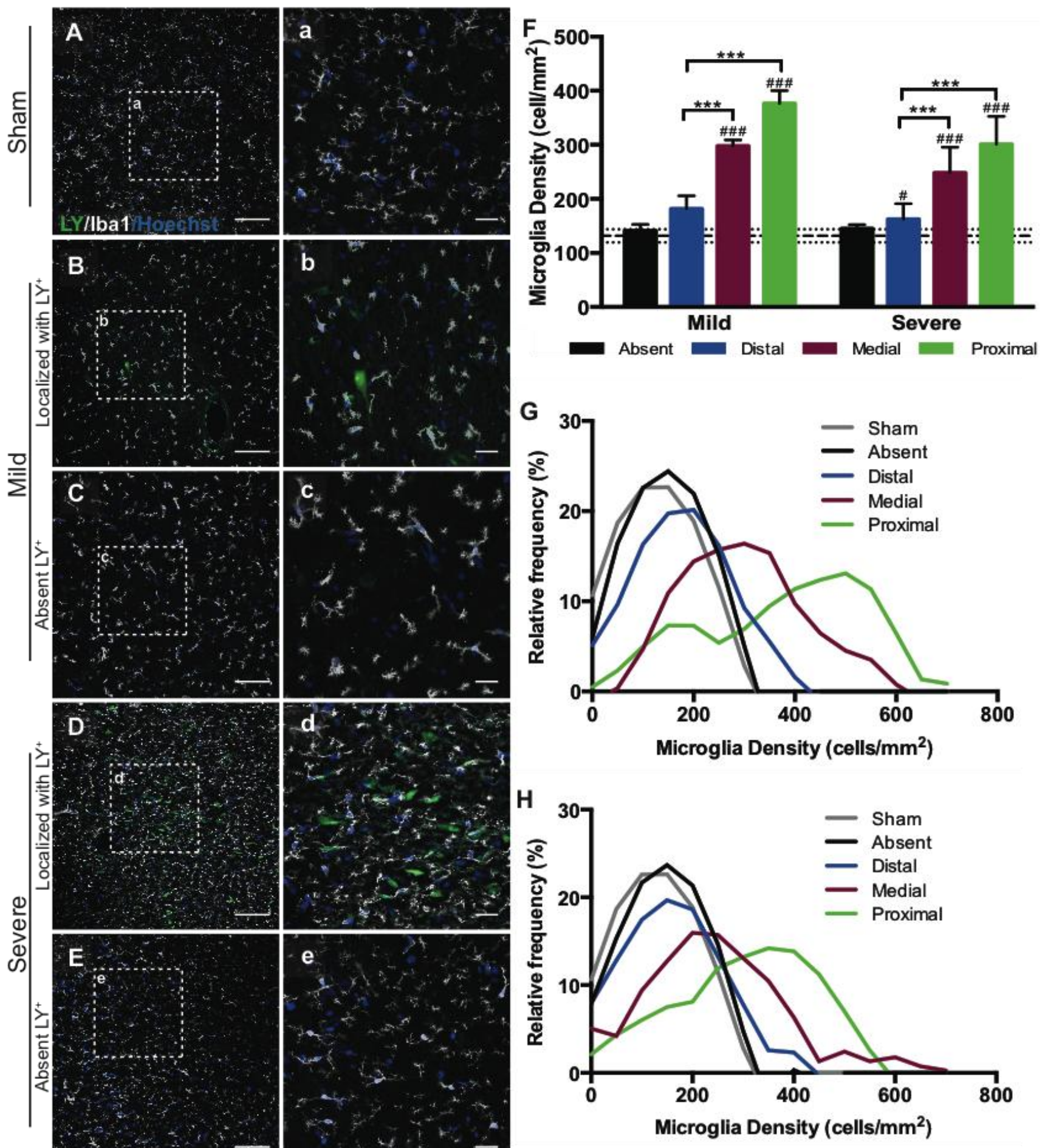

Figure 4. Cortical microglia incrementally increase in density in regions proximal to $\mathrm{LY}^{+}$ neurons post-TBI, but not in regions absent $\mathrm{LY}^{+}$neurons. Micrographs of $\mathrm{Hoechst}^{+}$(blue) and microglia (Iba1 ${ }^{+}$, white) spatial distribution around $\mathrm{LY}^{+}$neurons (green; A-E) with enlarged callout boxes (a-e). Images are taken from sham (A), mild (B-C), or severe (D-E) injuries in regions localized with $\mathrm{LY}^{+}$neurons $(\mathrm{B}, \mathrm{D})$ or in regions absent $\mathrm{LY}^{+}$neurons $(\mathrm{C}, \mathrm{E})$. Scale bars $=100 \mu \mathrm{m}$ (A-E) or scale bars $=25 \mu \mathrm{m}$ (a-e). Quantification of microglia density in mild and severe injuries 
(F) where microglia shift towards a higher density in regions more proximal to acutely injured neurons. Dashed horizontal lines represent sham animals' microglia density (mean \pm SEM). Smoothed relative frequency of microglia density 15 minutes after mild $(\mathrm{G})$ or severe $(\mathrm{H})$ injury paradigm. Column graph depicts mean \pm SEM and $*$ denotes $\mathrm{p}<0.05$; $* *$ denotes $\mathrm{p}<0.01$, *** denotes $\mathrm{p}<0.001$, and \# is significance relative to the injury-matched non-damaged group (black bars).
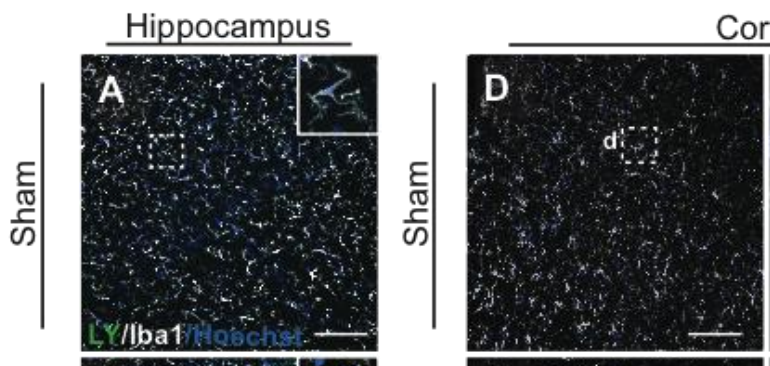

Cortex
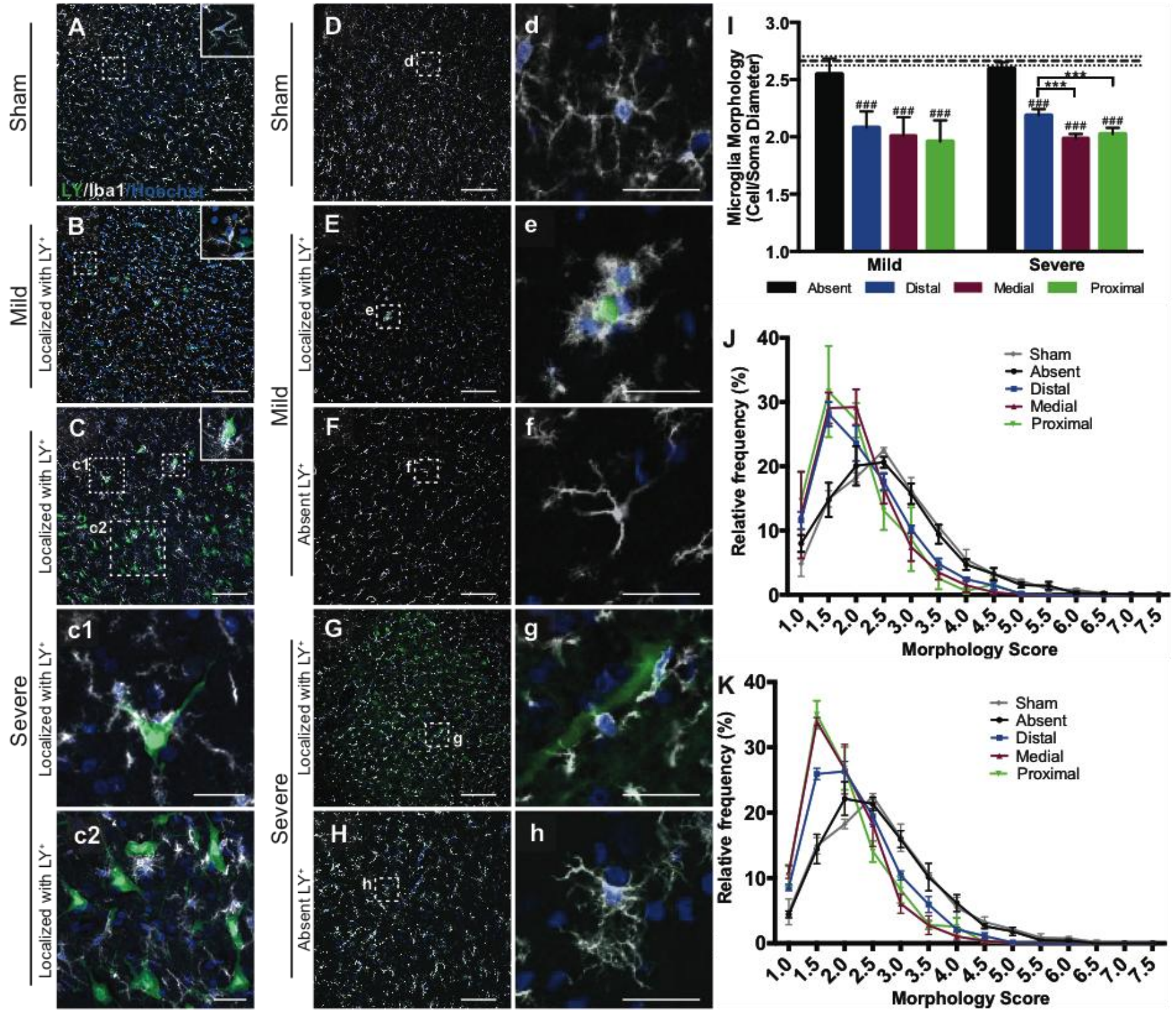

Figure 5. Microglia have more reactive morphologies only in regions localized with $\mathrm{LY}^{+}$ neurons post-TBI. Micrographs of $\mathrm{Hoechst}^{+}$(blue) and microglia (Iba1 ${ }^{+}$, white) morphology 
around $\mathrm{LY}^{+}$neurons (green; A-H) with enlarged call-out boxes (c-h). Images are taken from sham (A, D), mild (B, E-F), or severe $(\mathrm{C}, \mathrm{G}-\mathrm{H})$ animals in regions localized with $\mathrm{LY}^{+}$neurons $(\mathrm{B}-\mathrm{C}, \mathrm{E}$, $\mathrm{G})$ or regions absent $\mathrm{LY}^{+}$neurons $(\mathrm{F}, \mathrm{H})$. Scale bars $=100 \mu \mathrm{m}(\mathrm{A}-\mathrm{H})$ or scale bars $=25 \mu \mathrm{m}(\mathrm{c}-\mathrm{h})$. Single cell quantification of morphology in mild and severe injuries indicates a shift towards a more reactive morphology in regions localized around permeabilized neurons (I). Dashed horizontal lines represent sham animals (mean \pm SEM). Relative frequency of microglia morphology scores after mild (J) or severe (K) TBI. All graphs depict mean \pm SEM where * denotes $\mathrm{p}<0.05$; $* *$ denotes $\mathrm{p}<0.01$, $* * *$ denotes $\mathrm{p}<0.001$, and \# is significance relative to the injury-matched non-damaged group (black bars).

In tandem with our analysis of microglia density, we investigated changes in morphology in individual microglia. This analysis revealed that microglia morphology was not significantly different between sham animals and regions absent $\mathrm{LY}^{+}$neurons following either mild or severe TBI (Fig. 5I). However, in regions close to permeabilized neurons following either mild or severe TBI, there was a shift toward a more reactive morphology (Fig. 5I-K, Supplementary Fig. 2) although these reactive behaviors were not different between injury severities. Frankly amoeboid microglia were primarily observed in fields containing traumatically permeabilized neurons; indeed, activated microglia were often observed directly abutting and wrapping around $\mathrm{LY}^{+}$neurons (Fig. 5C, E, G). Overall, our single cell analysis revealed that $54 \%$ of microglia exhibited a reactive morphology score of $\leq 2.0$ (arbitrary threshold for analysis purposes) around permeabilized neurons post-TBI, as compared to only $28 \%$ of microglia in sham and $32 \%$ of microglia in regions without permeabilized neurons post-TBI $[\mathrm{p}<0.001$ : regions with $\mathrm{LY}^{+}$neurons post-TBI vs. sham or regions absent $\mathrm{LY}^{+}$neurons; $\mathrm{p}=0.3157$ (not significant): regions absent $\mathrm{LY}^{+}$neurons post-TBI vs. sham] (Supplementary Fig. 2; red lines).

In contrast to sham and single injuries, administration of repetitive injuries on the same day influenced microglia morphology to become even more reactive, with some microglia exhibiting an amoeboid morphology and some microglia exhibiting morphologies between hypertrophic and amoeboid states (Fig. 6A-B). Repetitive mild injuries on the same day resulted in less reactive microglia relative to repetitive severe injuries on the same day - which exhibited atypical, but clearly reactive microglia (Fig. 6A-B). After repetitive injuries separated by 7 days, microglia were still processesbearing, but the branches were often thicker and less numerous than sham, single injuries, and same-day repetitive injuries, suggesting further elevation of reactivity levels (Fig. 6C-D). Multiple amoeboid microglia were observed around $\mathrm{LY}^{+}$neurons after severe repetitive injuries separated by 7 days (Fig. 6D). These observations suggest that neuroinflammatory effects are exacerbated after repetitive closed- 
head diffuse brain injury, and should therefore be a consideration when investigating repetitive mild and/or severe TBI events.
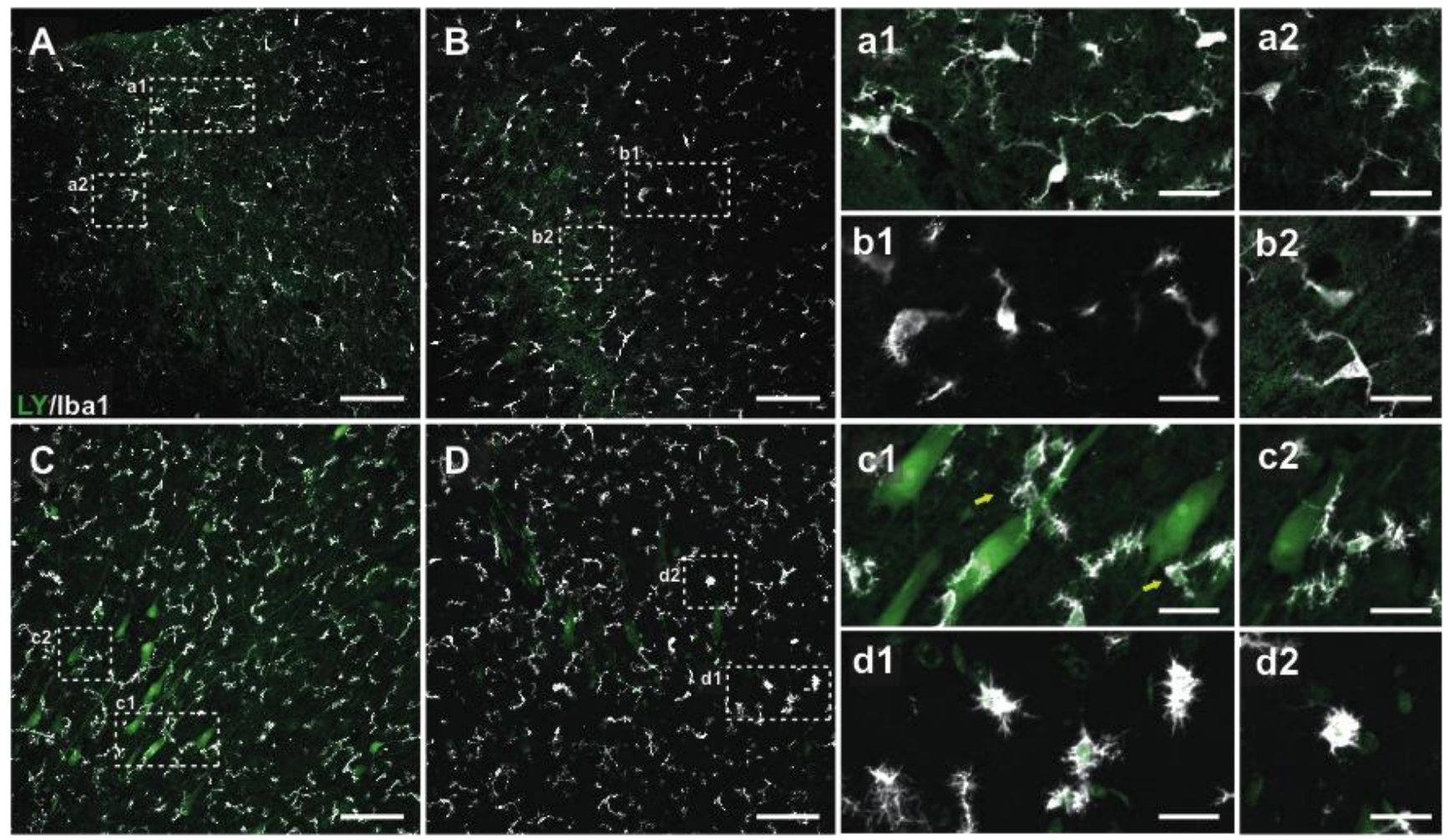

Figure 6. Microglia exhibit exacerbated activation states after repetitive TBI. Examples of microglia ( $\mathrm{Iba}^{+}$, white) reactivity around $\mathrm{LY}^{+}$neurons (green) after repetitive TBI separated by 15 minutes or separated by 7 days. Repetitive mild injuries separated by 15 minutes show microglia with variable morphologies (A), magnified in (a1, a2). Repetitive severe injuries separated by 15 minutes show microglia with atypical but reactive morphologies (B), magnified in (b1, b2). Repetitive mild injuries separated by 7 days showed extensive $\mathrm{LY}^{+}$permeability with microglia in partially activated state (C), magnified in (c1, c2; yellow arrows) with microglia in close proximity to $\mathrm{LY}^{+}$neurons. Repetitive severe injuries separated by 7 days showed partial $\mathrm{LY}^{+}$permeability with amoeboid or activated microglia (D) magnified in (d1, d2). Scale bars = $100 \mu \mathrm{m}(\mathrm{A}-\mathrm{D})$ or scale bars $=25 \mu \mathrm{m}(\mathrm{a}-\mathrm{d})$. 


\section{Discussion}

In this study, we assessed features of acute microglial activation after closed-head TBI in swine, since neuroinflammation is suspected to be a major contributor of progressive neurotoxicity, neurodegeneration, and neurological deficits after injury (Erturk et al., 2016; Patterson and Holahan, 2012). We found that neurons exhibiting acute plasma membrane disruptions were present in a multifocal pattern throughout the cerebral cortex, subcortical white matter, and hippocampal regions after either mild or severe injury, but with a significantly higher burden following severe TBI. Following mild or severe TBI, we observed microglia reactivity increased only in regions that contained permeabilized neurons, while microglia reactivity was unchanged in regions absent neuronal damage. Interestingly, we did not find significant differences in microglia density or morphology within fields containing permeabilized neurons after mild compared to severe TBI. However, it is important to note that our single cell quantification methodology does not directly account for the observation that the total burden of cortical neuronal permeability was greater following severe versus mild TBI. In fact, our single-cell analysis methodology was deliberately focused on areas exhibiting $\mathrm{LY}^{+}$neurons, and while providing a detailed examination of localized microglia reactivity, may not provide a representative measure of total microglia reactivity. Therefore, these findings must be interpreted contextually with the observation that there was a greater burden of neuronal permeability, and hence more regions of significant microglial activation, following severe versus mild TBI. Importantly, these data suggest that the presence of damaged neurons, as denoted by plasmalemmal disruptions, induced a profound yet highly localized impact on microglia reactivity within 15 minutes post-injury. However, failure to detect differences between localized microglial reactivity following mild versus severe TBI may also suggest that microglia are maximally responding to neuronal damage at this acute time; for instance this may suggest that it does not require a large "dose" of permeabilized neurons to elicit a maximal localized response within 15 minutes of trauma. In future studies, an examination of additional acute and sub-acute time points will be essential to determine if there is a divergence in localized microglial activation profiles between severe and mild closed-head diffuse TBI over time. We also speculate that globally measurable changes in microglial reactivity may manifest over longer time scales.

Increasing microglia densities in regions proximal to damaged neurons suggest that microglia could be migrating toward the damaged neurons or proliferating around damaged neurons. Alternatively, monocyte-derived macrophages could be infiltrating through the peripheral vasculature. Surprisingly, the microglia density measurements increased in proximity to injured neurons but did not change in regions distal to $\mathrm{LY}^{+}$neurons. These data imply that microglia were not migrating toward damaged 
neurons as this would have depleted microglial density in distal regions. While our analysis cannot exclude the possibility of either proliferation or recruitment, it is unlikely that microglia have the capacity to mitotically divide in such an acute time (Mander et al., 2006; Shankaran et al., 2007), suggesting that our Iba1 labeling may have captured a mix of endogenous microglia and rapidly infiltrating leukocytes, although further analysis will be required to differentiate these populations. Delineation between monocyte-derived macrophages and microglia-derived macrophages typically utilizes flow cytometry against CD-45 intensity (Wattananit et al., 2016). However, this procedure is not applicable in the current experimental paradigm because the premise of our analysis is microglial activation based on spatial proximity to permeabilized $\left(\mathrm{LY}^{+}\right)$neurons, a feature obviously lost during tissue dissociation required for flow cytometry. Future studies that are able to preserve spatial resolution of the tissue and identify origins of these $\mathrm{Iba}^{+}$cells may employ techniques such as in situ hybridization, monocyte-derived macrophage pre-labeling studies, and/or optimization of species (pig)specific antibodies; however, these techniques are non-trivial and non-standard (especially in the pig), and require extensive development and validation in order to reliably ascertain the origin and functionality of these cells.

This is the first report of rapid microglial activation following closed-head diffuse TBI in a large, gyrencephalic animal model and, to our knowledge, it is also the first report of reactive microglia preferentially localized to membrane-permeabilized neurons. Recent investigations of microglial behavior after rodent models of closed-head TBI suggest that microglia change morphology, may prevent parenchymal cell death within the first 12 hours after injury, and colocalize with debris escaping from leaky vasculature (Huber et al., 2016; Roth et al., 2014). Building on these findings, we utilized a single-cell analysis methodology and observed a shift toward a more reactive, amoeboid morphology in microglia localized to damaged neurons but did not observe reactive microglia morphology in regions absent neuronal damage. The profound shift of microglial morphology in regions containing neuronal damage underscores the seemingly direct influence of injured neurons on rapid microglial activation in our model. Indeed, as global injury-induced changes in microglial activation were not observed at this acute timepoint, our findings suggest that localized neuronal damage - such as plasmalemmal compromise - may be essential in driving acute microglial activation. Additionally, because LY was administered intraventricularly and evenly distributed throughout the brain parenchyma prior to TBI, we do not believe that blood brain barrier disruption would influence the access of membrane-damaged neurons to LY. However, local tissue deformations/strain fields that induce neuronal membrane damage may also colocalize with regions of mechanically induced blood brain barrier compromise. Therefore, 
disruption of the blood brain barrier may also contribute to the observed acute microglial reactivity, and future studies using this porcine model should address this issue.

We also examined the effects of repetitive diffuse brain injury on acute microglial activation in relation to traumatically permeabilized neurons. Because administration of repetitive injures confounds evaluation of "immediate" microglial activation, detailed single-cell quantitative analyses of microglial distribution and morphology were not performed in this cohort. However, our qualitative findings suggest that, not surprisingly, microglia become more reactive and amoeboid following repetitive injuries relative to single injury. These augmented microglial reactivity changes were most striking following repetitive injuries separated by 7 days, suggesting that secondary injury progression may significantly contribute to the development of microgliosis and/or an increased microglia sensitivity to subsequent injuries in this model. Interestingly, in the repetitive cohort, microglia reactivity did not appear to correlate as strongly with the presence (or density) of $\mathrm{LY}^{+}$cells, as was demonstrably the case with single injuries. These findings suggest that repetitive injury and/or a longer time point after a first injury may elicit more global changes in microglial reactivity; however, additional experiments, including more experimental time points, will be required to substantiate this conclusion. Regardless, these findings of exacerbated microglial activation following repetitive TBI may have future implications for intermediate and chronic neurodegenerative sequelae, potentially including chronic traumatic encephalopathy.

Collectively, these findings demonstrate rapid microglial activation - in particular an increase in the density of amoeboid morphologies - following closed-head diffuse TBI, with traumatically permeabilized neurons serving as localized epicenters of microglia reactivity in the acute phase. Although multiple neuronal and axonal injury phenotypes have been described post-TBI (Johnson et al., 2016; Singleton and Povlishock, 2004; Stone et al., 2004), the population exhibiting plasma membrane damage may be particularly powerful in driving neuroinflammatory responses due to the uncontrolled release and subsequent diffusion of factors normally sequestered intracellularly. Although the modified Hovens ratio that we employed to quantitatively assess microglia diameter was capable of detecting microglia reactivity, this methodology may not be sensitive to more subtle morphological changes such as alterations in secondary process branching. As such, future studies may build on this work by employing more comprehensive morphological analyses on a per-cell basis to better detect other reactive changes such as secondary process thickening, expansion, or retraction - such nuanced alterations may also be sensitive to neuronal trauma or other TBI effect. Further investigation into the contribution of these rapidly activated microglia to neuronal stability after TBI is necessary to fully appreciate their role in pathophysiological progression. Activated microglia in the vicinity of acutely 
injured neurons may be beneficial to reestablish neuronal homeostasis, sequester harmful molecules, or release acute neuroprotective cytokines (Herx et al., 2000; Roth et al., 2014). However, it is also possible that acutely activated microglia hinder the health of permeabilized neurons by secreting deleterious factors - cytokines that could non-specifically distress neurons not originally damaged by the traumatic event (Block et al., 2007; Scherbel et al., 1999). Indeed, neuroinflammation contributes to neuronal regeneration, but has also been suggested to have a leading role in chronic neural dysfunction and degeneration (Patterson and Holahan, 2012). Importantly, our findings were attained using a gyrencephalic animal model that closely replicates the injury biomechanics of TBI in humans. This suggests that acute inflammatory pathways may be relevant even following so-called "mild" TBI in humans, therefore future efforts should be directed towards the goal of augmenting potential protective effects while preventing the amplification of deleterious signaling.

\section{Conclusion}

It is well established that microglia-induced inflammation significantly contributes to pathological progression after CNS injury. However, understanding the onset, mechanism(s), and implications of inflammation in the brain is essential to develop successful and translatable immunomodulatory therapeutics for concussion and more severe TBIs. The present findings suggest that acute neuronal damage - manifested via plasma membrane disruptions - may be essential for rapid microglial activation following closed-head diffuse TBI. Indeed, we found that within minutes postinjury, activated microglia were appreciable in the vicinity of injured neurons, but global changes were not detectable, suggesting that neuronal damage is an initial "trigger" for neuroinflammatory responses in non-penetrating TBI. This platform may be further exploited to investigate the influence of the innate immune system on neuronal homeostasis and/or pathology following closed-head diffuse TBI.

\section{Acknowledgments}

The authors thank Dr. Victoria Johnson and Carolyn Keating for their technical assistance and guidance. This work was made possible through financial support provided by the Department of Veterans Affairs (RR\&D Merit Review \#B1097-I), the National Institutes of Health (NINDS T32NS043126; NICHD U54-HD086984), and Penn's University Research Foundation. K.L. Wofford was supported by a fellowship from the National Science Foundation STEM GK-12 program "Catalyzing STEM Education via the NAE Engineering Grand Challenges”, grant \#DGE-0947936 through Drexel 
University. None of the funding sources aided in the collection, analysis, and interpretation of data, in the writing of the report, or in the decision to submit the paper for publication. The authors have no conflicts of interest related to this work to disclose. 


\section{References}

Adams, J.H., Doyle, D., Ford, I., Gennarelli, T.A., Graham, D.I., McLellan, D.R., 1989. Diffuse axonal injury in head injury: definition, diagnosis, and grading. Histopathology 15, 49-59. doi:10.1017/CBO9781107415324.004

Block, M.L., Zecca, L., Hong, J.-S., 2007. Microglia-mediated neurotoxicity: uncovering the molecular mechanisms. Nat. Rev. Neurosci. 8, 57-69. doi:10.1038/nrn2038

Browne, K.D., Chen, X.-H., Meaney, D.F., Smith, D.H., 2011. Mild traumatic brain injury and diffuse axonal injury in swine. J. Neurotrauma 28, 1747-1755. doi:10.1089/neu.2011.1913

Coronado, V.G., McGuire, L.C., Sarmiento, K., Bell, J., Lionbarger, M.R., Jones, C.D., Geller, A.I., Khoury, N., Xu, L., 2012. Trends in Traumatic Brain Injury in the U.S. and the public health response: 1995-2009. J. Safety Res. 43, 299-307. doi:10.1016/j.jsr.2012.08.011

Cullen, D.K., Harris, J.P., Browne, K.D., Wolf, J.A., Duda, J.E., Meaney, D.F., Margulies, S.S., Smith, D.H., 2016. A porcine model of traumatic brain injury via head rotational acceleration. Methods Mol. Biol. 1462, 289-324.

Cullen, D.K., Vernekar, V.N., LaPlaca, M.C., 2011. Trauma-Induced Plasmalemma Disruptions in Three-Dimensional Neural Cultures Are Dependent on Strain Modality and Rate. J. Neurotrauma 28, 2219-2233. doi:10.1089/neu.2011.1841

Davalos, D., Grutzendler, J., Yang, G., Kim, J. V, Zuo, Y., Jung, S., Littman, D.R., Dustin, M.L., Gan, W.-B., 2005. ATP mediates rapid microglial response to local brain injury in vivo. Nat. Neurosci. 8, 752-758. doi:10.1038/nn1472

De Kruijk, J.R., Twijnstra, A., Leffers, P., Twinjstra, A., Leffers, P., 2001. Diagnostic criteria and differential diagnosis of mild traumatic brain injury. Brain Inj. 15, 999-106.

De Monte, V.E., Geffen, G.M., Massavelli, B.M., 2006. The effects of post-traumatic amnesia on information processing following mild traumatic brain injury. Brain Inj. 20, 1345-1354. doi:10.1080/02699050601082073

Dibaj, P., Nadrigny, F., Steffens, H., Scheller, A., Hirrlinger, J., Schomburg, E.D., Neusch, C., Kirchhoff, F., 2010. NO mediates microglial response to acute spinal cord injury under ATP control in vivo. Glia 58, 1133-1144. doi:10.1002/glia.20993

Erturk, A., Mentz, S., Stout, E.E., Hedehus, M., Dominguez, S.L., Neumaier, L., Krammer, F., Llovera, G., Srinivasan, K., Hansen, D. V., Liesz, A., Scearce-Levie, K.A., Sheng, M., 2016. Interfering with the Chronic Immune Response Rescues Chronic Degeneration After Traumatic Brain Injury. J. Neurosci. 36, 9962-9975. doi:10.1523/JNEUROSCI.1898-15.2016 
Farkas, O., Lifshitz, J., Povlishock, J.T., 2006. Mechanoporation Induced by Diffuse Traumatic Brain Injury: An Irreversible or Reversible Response to Injury? J. Neurosci. 26, 3130-3140. doi:10.1523/JNEUROSCI.5119-05.2006

Faul M, Xu L, Wald MM, C.V., 2010. Traumatic brain injury in the United States: emergency department visits, hospitalizations, and deaths. Centers Dis. Control Prev. Natl. Cent. Inj. Prev. Control 891-904. doi:10.1016/B978-0-444-52910-7.00011-8

Fitzmaurice, G.M., Laird, N.M., Ware, J.H., 2004. Applied longitudinal analysis. John Wiley \& Sons. Geddes, D.M., Cargill, R.S., LaPlaca, M.C., 2003. Mechanical stretch to neurons results in a strain rate and magnitude-dependent increase in plasma membrane permeability. J. Neurotrauma 20, 10391049. doi:10.1089/089771503770195885

Harris, J.P., 2015. Traumatically induced neuronal plasmalemmal permeability and dendritic beading in the hippocampus following diffuse brain injury in swine. J Neurotrauma (conference proceedings) 32, A-1-A-152.

Herx, L.M., Rivest, S., Yong, V.W., 2000. Central nervous system-initiated inflammation and neurotrophism in trauma: IL-1 beta is required for the production of ciliary neurotrophic factor. $\mathrm{J}$. Immunol. 165, 2232-2239. doi:10.4049/jimmunol.165.4.2232

Hovens, I., Nyakas, C., Schoemaker, R., 2014. A novel method for evaluating microglial activation using ionized calcium-binding adaptor protein-1 staining: cell body to cell size ratio.

Neuroimmunol. Neuroinflammation 1, 82-88. doi:10.4103/2347-8659.139719

Huber, B.R., Meabon, J.S., Hoffer, Z.S., Zhang, J., Hoekstra, J.G., Pagulayan, K.F., McMillan, P.J., Mayer, C.L., Banks, W.A., Kraemer, B.C., Raskind, M.A., McGavern, D.B., Peskind, E.R., Cook, D.G., 2016. Blast exposure causes dynamic microglial/macrophage responses and microdomains of brain microvessel dysfunction. Neuroscience 319, 206-220.

doi:10.1016/j.neuroscience.2016.01.022

Hyder, A. a, Wunderlich, C. a, Puvanachandra, P., Gururaj, G., Kobusingye, O.C., 2007. The impact of traumatic brain injuries: a global perspective. NeuroRehabilitation 22, 341-353. doi:http://iospress.metapress.com/content/103177/?sortorder=asc

Johnson, V.E., Stewart, J.E., Begbie, F.D., Trojanowski, J.Q., Smith, D.H., Stewart, W., 2013. Inflammation and white matter degeneration persist for years after a single traumatic brain injury. Brain 136, 28-42. doi:10.1093/brain/aws322

Johnson, V.E., Stewart, W., Weber, M.T., Cullen, D.K., Siman, R., Smith, D.H., 2016. SNTF immunostaining reveals previously undetected axonal pathology in traumatic brain injury. Acta Neuropathol. 131, 115-135. doi:10.1007/s00401-015-1506-0 
Kelley, B.J., Lifshitz, J., Povlishock, J.T., 2007. Neuroinflammatory responses after experimental diffuse traumatic brain injury. J. Neuropathol. Exp. Neurol. 66, 989-1001. doi:10.1097/NEN.0b013e3181588245

Lafrenaye, A.D., Todani, M., Walker, S.A., Povlishock, J.T., 2015. Microglia processes associate with diffusely injured axons following mild traumatic brain injury in the micro pig. J.

Neuroinflammation 12, 186-201. doi:10.1186/s12974-015-0405-6

Langlois, J. a, Rutland-Brown, W., Wald, M.M., 2006. The epidemiology and impact of traumatic brain injury: a brief overview. J. Head Trauma Rehabil. 21, 375-8. doi:00001199-200609000-00001 [pii]

LaPlaca, M.C., Lee, V.M., Thibault, L.E., 1997. An in vitro model of traumatic neuronal injury: loading rate-dependent changes in acute cytosolic calcium and lactate dehydrogenase release. J.

Neurotrauma 14, 355-68. doi:10.1089/neu.1997.14.355

LaPlaca, M.C., Prado, G.R., Cullen, D.K., Simon, C.M., 2009. Plasma membrane damage as a marker of neuronal injury. Conf. Proc. IEEE Eng. Med. Biol. Soc. 2009, 1113-1116. doi:10.1109/IEMBS.2009.5334457

Lee, M., 2013. Neurotransmitters and microglial-mediated neuroinflammation. Curr. Protein Pept. Sci. 14, 21-32. doi:10.2174/1389203711314010005

Leininger, B.E., Gramling, S.E., Farrell, a D., Kreutzer, J.S., Peck, E. a, 1990. Neuropsychological deficits in symptomatic minor head injury patients after concussion and mild concussion. J. Neurol. Neurosurg. Psychiatry 53, 293-296. doi:10.1136/jnnp.53.4.293

Mander, P.K., Jekabsone, A., Brown, G.C., 2006. Microglia proliferation is regulated by hydrogen peroxide from NADPH oxidase. J. Immunol. 176, 1046-1052. doi:10.4049/jimmunol.176.2.1046

Meaney, D.F., Smith, D.H., Shreiber, D.I., Bain, A.C., Miller, R.T., Ross, D.T., Gennarelli, T.A., 1995. Biomechanical Analysis of Experimental Diffuse Axonal Injury. J. Neurotrauma 12.

Ommaya, A.K., Gennarelli, T.A., 1974. Cerebral concussion and traumatic unconsciousness. Correlation of experimental and clinical observations of blunt head injuries. J. Neurol. 97, 633654.

Patterson, Z.R., Holahan, M.R., 2012. Understanding the neuroinflammatory response following concussion to develop treatment strategies. Front. Cell. Neurosci. 6, 1-10. doi:10.3389/fncel.2012.00058

Povlishock, J.T., 1992. Traumatically induced axonal injury: pathogenesis and pathobiological implications. Brain Pathol. 2, 1-12.

Roth, T.L., Nayak, D., Atanasijevic, T., Koretsky, A.P., Latour, L.L., McGavern, D.B., 2014. Transcranial amelioration of inflammation and cell death after brain injury. Nature 505, 223-8. 
doi:10.1038/nature 12808

Scherbel, U., Raghupathi, R., Nakamura, M., Saatman, K.E., Trojanowski, J.Q., Neugebauer, E., Marino, M.W., Mcintosh, T.K., 1999. Differential acute and chronic responses of tumor necrosis factor-deficient mice to experimental brain injury. Neurobiology 96, 8721-8726. doi:10.1073/pnas.96.15.8721

Shankaran, M., Marino, M.E., Busch, R., Keim, C., King, C., Lee, J., Killion, S., Awada, M., Hellerstein, M.K., 2007. Measurement of Brain Microglial Proliferation Rates In Vivo in Response to Neuroinflammatory Stimuli: Application to Drug Discovery. J. Neurosci. Res. 85, 2352-2359. doi:10.1002/jnr

Simon, C.M., Sharif, S., Tan, R.P., LaPlaca, M.C., 2009. Spinal cord contusion causes acute plasma membrane damage. J. Neurotrauma 26, 563-574.

Singleton, R.H., Povlishock, J.T., 2004. Identification and Characterization of Heterogeneous Neuronal Injury and Death in Regions of Diffuse Brain Injury: Evidence for Multiple Independent Injury Phenotypes. J. Neurosci. 24, 3543-3553. doi:10.1523/JNEUROSCI.5048-03.2004

Smith, D.H., Chen, X.-H., Xu, B.-N., McIntosh, T.K., Gennarelli, T.A., Meaney, D.F., 1997.

Characterization of diffuse axonal pathology and selective hippocampal damage following inertial brain trauma in the pig. J. Neuropathol. Exp. Neurol. 56, 822-834.

Smith, D.H., Meaney, D.F., 2000. Axonal Damage in Traumatic Brain Injury. Neurosci. 6, 483-495. doi:10.1177/107385840000600611

Smith, D.H., Nonaka, M., Miller, R., LEONI, M., Chen, X.-H.H., ALSOP, D., MEANEY, D.F., 2000. Immediate coma following inertial brain injury dependent on axonal damage in the brainstem. J. Neurosurg. 93, 315-322.

Stone, J.R., Okonkwo, D.O., Dialo, A.O., Rubin, D.G., Mutlu, L.K., Povlishock, J.T., Helm, G.A., 2004. Impaired axonal transport and altered axolemmal permeability occur in distinct populations of damaged axons following traumatic brain injury. Exp. Neurol. 190, 59-69. doi:10.1016/j.expneurol.2004.05.022

The R Development Core Team, 2016. R: A Language and Environment for Statistical Computing. R Found. Stat. Comput. doi:10.1038/sj.hdy.6800737

Wattananit, S., Tornero, D., Graubardt, N., Memanishvili, T., Monni, E., Tatarishvili, J., Miskinyte, G., Ge, R., Ahlenius, H., Lindvall, O., Schwartz, M., Kokaia, Z., 2016. Monocyte-Derived Macrophages Contribute to Spontaneous Long-Term Functional Recovery after Stroke in Mice. J. Neurosci. 36, 4182-4195. doi:10.1523/JNEUROSCI.4317-15.2016

Weber, J.T., Rzigalinski, B.A., Willoughby, K.A., Moore, S.F., Ellis, E.F., 1999. Alterations in calcium- 
mediated signal transduction after traumatic injury of cortical neurons. Cell Calcium 26, 289-99. doi:10.1054/ceca.1999.0082

Whalen, M.J., Dalkara, T., You, Z., Qiu, J., Bermpohl, D., Mehta, N., Suter, B., Bhide, P.G., Lo, E.H., Ericsson, M., Moskowitz, M. a, 2008. Acute plasmalemma permeability and protracted clearance of injured cells after controlled cortical impact in mice. J. Cereb. Blood Flow Metab. 28, 490-505. doi:10.1038/sj.jcbfm.9600544

Wolf, J.A., Koch, P.F., 2016. Disruption of Network Synchrony and Cognitive Dysfunction After Traumatic Brain Injury. Front. Syst. Neurosci. 10, 1-14. doi:10.3389/FNSYS.2016.00043 